\title{
The inhibitory role of recombinant P-selectin glycoprotein ligand immunoglobulin $G$ on portal vein thrombosis based on a novel rat model
}

\author{
YUNHAI WEI $^{1}$, JIE SHAO $^{2}$, HUA SHEN $^{1}$, YAN WANG $^{1}$, GUOLIANG CAO $^{1}$, \\ WENXIAN CHEN ${ }^{3}$, JINYU ZHANG ${ }^{1}$ and LEI YIN ${ }^{4}$ \\ ${ }^{1}$ Surgical Department, Huzhou Central Hospital, Zhejiang University Huzhou Hospital; \\ ${ }^{2}$ Integrated Traditional Chinese and Western Medicine Surgery, Huzhou Maternal and Child Health Care Hospital; \\ ${ }^{3}$ Ultrasonography Department and ${ }^{4}$ Department of Gastrointestinal Surgery, Huzhou Central Hospital, \\ Zhejiang University Huzhou Hospital, Huzhou, Zhejiang 313000, P.R. China
}

Received March 21, 2018; Accepted December 27, 2018

DOI: $10.3892 /$ etm.2019.7394

\begin{abstract}
The current study aimed to reveal a novel method for constructing a portal vein thrombosis (PVT) model in rats and to evaluate the inhibitory role of recombinant $\mathrm{P}$-selectin glycoprotein ligand immunoglobulin G (rPSGL-Ig) on the formation of PVT. The PVT model was constructed in rats through intermittent portal vein obstruction (IPVO) combined with endangium destruction. A total of $4 \mathrm{mg} / \mathrm{kg}$ rPSGL-Ig was intraperitoneally pre-injected into rats $1 \mathrm{~h}$ prior to model construction. Changes in the thrombus size and vessel diameter were observed by B-scan ultrasonography. Histopathological changes in the portal vein, central hepatic vein and vasa intestini tenuis were observed using hematoxylin and eosin staining. Additionally, histopathological changes in the portal vein were observed by transmission electron microscopy. A total of $8 \mathrm{mg} / \mathrm{kg}$ rPSGL-Ig or $2 \times 10^{4} \mathrm{U} / \mathrm{kg}$ urokinase were used to compare the thrombolytic effects and thrombus sizes. The PVT model was successfully constructed in rats, and exhibited a significantly greater thrombus size and vessel diameter compared with the control group $(\mathrm{P}<0.05)$. Intervention with rPSGL-Ig significantly inhibited the formation of PVT, and resulted in a significantly lower thrombus size and vessel diameter compared with the model group $(\mathrm{P}<0.05)$. Additionally,
\end{abstract}

Correspondence to: Dr Yunhai Wei, Surgical Department, Huzhou Central Hospital, Zhejiang University Huzhou Hospital, 198 Hongqi Road, Huzhou, Zhejiang 313000, P.R. China

E-mail: yunnyw_1008@126.com

Abbreviations: PVT, portal vein thrombosis; rPSGL-Ig, recombinant P-selectin glycoprotein ligand immunoglobulin G; TEM, transmission electron microscope; URO, urokinase

Key words: portal vein thrombosis, model, recombinant P-selectin glycoprotein ligand immunoglobulin $\mathrm{G}$, histopathology, B-scan ultrasonography histopathological changes in the portal vein, central hepatic vein and vasa intestini tenuis in PVT rats were considerably reversed following the intervention with rPSGL-Ig. rPSGL-Ig demonstrated a lower thrombolytic effect compared with that of URO. IPVO combined with endangium destruction effectively constructed a PVT model in rats. rPSGL-Ig effectively prevented PVT in rats. rPSGL-Ig may be used in future studies for the treatment of patients with PVT.

\section{Introduction}

Portal vein thrombosis (PVT) is a form of venous thrombus, which develops into the trunk of the portal vein and may extend to the splenic or superior mesenteric veins (1). PVT occurs in association with pancreatitis, cirrhosis, diverticulitis and malignancy, and is known as a serious complication of splenectomy $(2,3)$. It has been reported that the prevalence of PVT is $4.4-15 \%$ in patients with cirrhosis, approximately $35 \%$ in cirrhotic patients with hepatocellular carcinoma (4) and 37.5-43.5\% in patients who have undergone a splenectomy (5). As PVT is the main cause of portal hypertension, preventing PVT has become an important goal in clinical practice (6).

P-selectin, a cell adhesion molecule with procoagulant properties, serves an important role in thrombosis (7). An interaction between $\mathrm{P}$-selectin and P-selectin glycoprotein ligand (PSGL) has been demonstrated to induce the recruitment of neutrophils and macrophages to promote the generation of procoagulant microparticles (8). Recombinant (r)PSGL immunoglobulin G (Ig) is an antibody of PSGL-1 and antagonizes P-selectin by competing with PSGL-1 to inhibit thrombosis (9). It has been reported that following 14-day treatment with rPSGL-Ig, the percentage of spontaneous vein reopening in the proximal iliac vein of baboons with vein thrombi was significantly increased compared with that in the control group (62\% vs. $8 \%$, respectively) (10). The occurrence of thrombi in the jugular veins of cats was completely $(4.0 \mathrm{mg} / \mathrm{kg})$ or partially $(1.0 \mathrm{mg} / \mathrm{kg}$ ) prevented by rPSGL-Ig (11). rPSGL-Ig inhibits the binding of circulating activated platelets with neutrophils at damaged arterial surfaces (12). Although 
rPSGL-Ig is known to inhibit thrombosis, the specific roles of rPSGL-Ig in preventing PVT remain unclear.

Since the first vein thrombosis model was established in rats in 1980 by ligation of the inferior vena cava (13), additional models have been constructed using various methods. Complete vein ligation is a commonly used modeling method for vein thrombosis $(14,15)$. Although a stable thrombus can be formed, complete vein ligation is not suitable for dynamic observation of antithrombotic drugs because the patency of the venous proximal end cannot be guaranteed (16). Additionally, foreign bodies and electric stimulation cause great damage to veins, and the injection of a thrombus-inducing agent may influence the evaluation of antithrombotic drugs (17-19). As studies using PVT models are limited, a suitable modeling method of PVT is urgently needed.

In the current study, a novel modeling method, intermittent portal vein obstruction (IPVO) combined with endangium destruction, was used to construct a PVT model in rats. The effects of rPSGL-Ig on PVT formation were evaluated by B-scan ultrasonography, hematoxylin-eosin (HE) staining and transmission electron microscopy (TEM). The authors of the current study developed an effective method for constructing a PVT model in rats and determined the inhibitory role of rPSGL-Ig in PVT formation.

\section{Materials and methods}

Construction of the rat model of PVT. A total of 90 Sprague Dawley specific pathogen-free rats (weight, 200-300 g; age, 2-3 months; female, $n=45$; male, $n=45$ ) were purchased from Shanghai Jiesijie Laboratory Animal Co. Ltd. (Shanghai, China). The rats were housed at $26 \pm 1^{\circ} \mathrm{C}$ in a standalone environment under an alternating day and night cycle of 12/12 h with free access to water and food. Rats were made to fast for $24 \mathrm{~h}$ prior to model construction and the PVT model was constructed through IPVO combined with endangium destruction. The rats were anesthetized by an intraperitoneal injection of $5 \%$ chloral hydrate $(350 \mathrm{mg} / \mathrm{kg})$ and fixed on an operating table. The abdominal cavity was opened by making a longitudinal incision $(4-5 \mathrm{~cm})$ along the epigastric midline (Fig. 1A and B). The intestinal canal was removed from the abdominal cavity with a wet cotton swab and covered with gauze containing physiological saline (Fig. 1C). Connective tissue around the portal vein was bluntly separated and $1.5-2 \mathrm{~cm}$ of the free portal vein was isolated (Fig. 1D). Next, the proximal and distal ends of the free portal vein (including the epidural catheter) were ligated using a suture 4-0 (Fig. 1E). Toothless tweezers were used to clamp the portal vein at the proximal and distal ends for $5 \mathrm{sec}$ every $5 \mathrm{~min}$ (Fig. 1F and G). Portal vein ligation was stopped after 20 min of treatment and then blood flow was allowed for $10 \mathrm{~min}$. These steps (ligation and clamp) were repeated five times. Finally, $2 \mathrm{ml} \mathrm{3 \%}$ ceftazidime solution was injected into the abdominal cavity and the abdominal cavity was sutured (Fig. 1H). The formation of PVT (including the thrombus size and vessel diameter) was continuously observed prior to and following the surgery using a B-mode ultrasound instrument (Philips Healthcare, Amsterdam, The Netherlands). The sham operation group was used as a control; in this group the portal vein was only dissociated, and no ligation and clamping was performed.
Reverse transcription-quantitative polymerase chain reaction (RT-qPCR). RT-qPCR analyses were performed to detect the mRNA expression level of P-selectin, a biomarker of PVT (20) in rats of the model and control groups $24 \mathrm{~h}$ following surgery. Total RNA was extracted from blood samples using RNA Extraction kit and reverse transcribed into cDNA using the First Strand cDNA Synthesis kit (both Beyotime Institute of Biotechnology, Shanghai, China). The following thermocycling conditions were used for cDNA synthesis: $45^{\circ} \mathrm{C}$ for $60 \mathrm{~min}$ and $70^{\circ} \mathrm{C}$ for $10 \mathrm{~min}$. qPCR was subsequently performed using the BeyoFast ${ }^{\mathrm{TM}}$ SYBR Green qPCR Mix (2X) kit (Beyotime Institute of Biotechnology) using the following specific primer pairs: P-selectin forward, 5'-GAGGCAGAGACCTCA CAGCCAG-3' and reverse, 5'-GTCAGGTAAGTGGCCAAT G-3'; and $\beta$-actin forward, 5'-ACACCTTCTACAATGAGC TG-3' and reverse, 5'-CTGCTTGCTGATCCCATCT-3'. The following thermocycling conditions were used for qPCR: Initial denaturation at $94^{\circ} \mathrm{C}$ for $3 \mathrm{~min} ; 30$ cycles at $94^{\circ} \mathrm{C}$ for $30 \mathrm{sec}, 62^{\circ} \mathrm{C}$ for $30 \mathrm{sec}$ and $72^{\circ} \mathrm{C}$ for $30 \mathrm{sec}$; and a final extension at $72^{\circ} \mathrm{C}$ for $5 \mathrm{~min}$. The relative P-selectin mRNA levels were quantified using the $2^{-\Delta \Delta \mathrm{Cq}}$ method (21) and normalized to the internal reference gene $\beta$-actin.

rPSGL-Ig intervention. Rats were divided into three groups (10 rats/group): Model, Control and rPSGL-Ig. rPSGL-Ig was prepared by Hangzhou S-Evans Biosciences Co., Ltd. (Hangzhou, China). A total of $4 \mathrm{mg} / \mathrm{kg}$ rPSGL-Ig was intraperitoneally injected into PVT rats at $1 \mathrm{~h}$ after model construction (the rPSGL-Ig group). The formation of PVT in the rPSGL-Ig group was further evaluated by B-scan ultrasonography 6,12 and $24 \mathrm{~h}$ after the surgeries, and by HE staining and TEM.

HE staining and TEM. HE staining was performed on the portal vein, central hepatic vein and vasa intestinae tenuis of rats in different groups $24 \mathrm{~h}$ following surgery. Tissue samples were fixed in $2 \%$ paraformaldehyde overnight at $4^{\circ} \mathrm{C}$. Tissue samples were dehydrated by ascending ethanol series (50, 70, 80 and $90 \%$ ethanol each for $15 \mathrm{~min}, 70 \%$ ethanol overnight, and $100 \%$ ethanol for $20 \mathrm{~min}$ ), soaked in acetone twice for $15 \mathrm{~min}$ and embedded in Araldite. Following 48-h polymerization at $65^{\circ} \mathrm{C}$, the embedded tissue samples were cut into ultrathin slices $(70 \mathrm{~nm})$ using an ultramicrotome (EM UC7; Leica Microsystems GmbH, Wetzlar, Germany). Tissue sections were stained with hematoxylene for $4 \mathrm{~min}$ at room temperature and couter stained with eosin for $90 \mathrm{sec}$ at room temperature, and observed under a light microscope (YYS-190E; Shanghai Optical Instrument, Shanghai, China) at a magnification x100 and x400.

TEM were performed on the portal vein of rats in different groups. Tissue slices were prepared as described above. Following staining with uranyl acetate and lead citrate (both Sinopharm Chemical Reagent Co., Ltd., Beijing, China) each for $10 \mathrm{~min}$ at room temperature, the samples were observed by TEM (JEM-1230; JEOL, Ltd., Tokyo, Japan) 6, 12 and 24 h after the surgeries.

Evaluation of the thrombolytic effect of rPSGL-Ig. PVT model $\mathrm{SD}$ rats were divided randomly into four groups ( $\mathrm{n}=6 /$ group): Control, $4 \mathrm{mg} / \mathrm{kg}$ rPSGL-Ig, $6 \mathrm{mg} / \mathrm{kg}$ rPSGL-Ig and $8 \mathrm{mg} / \mathrm{kg}$ 

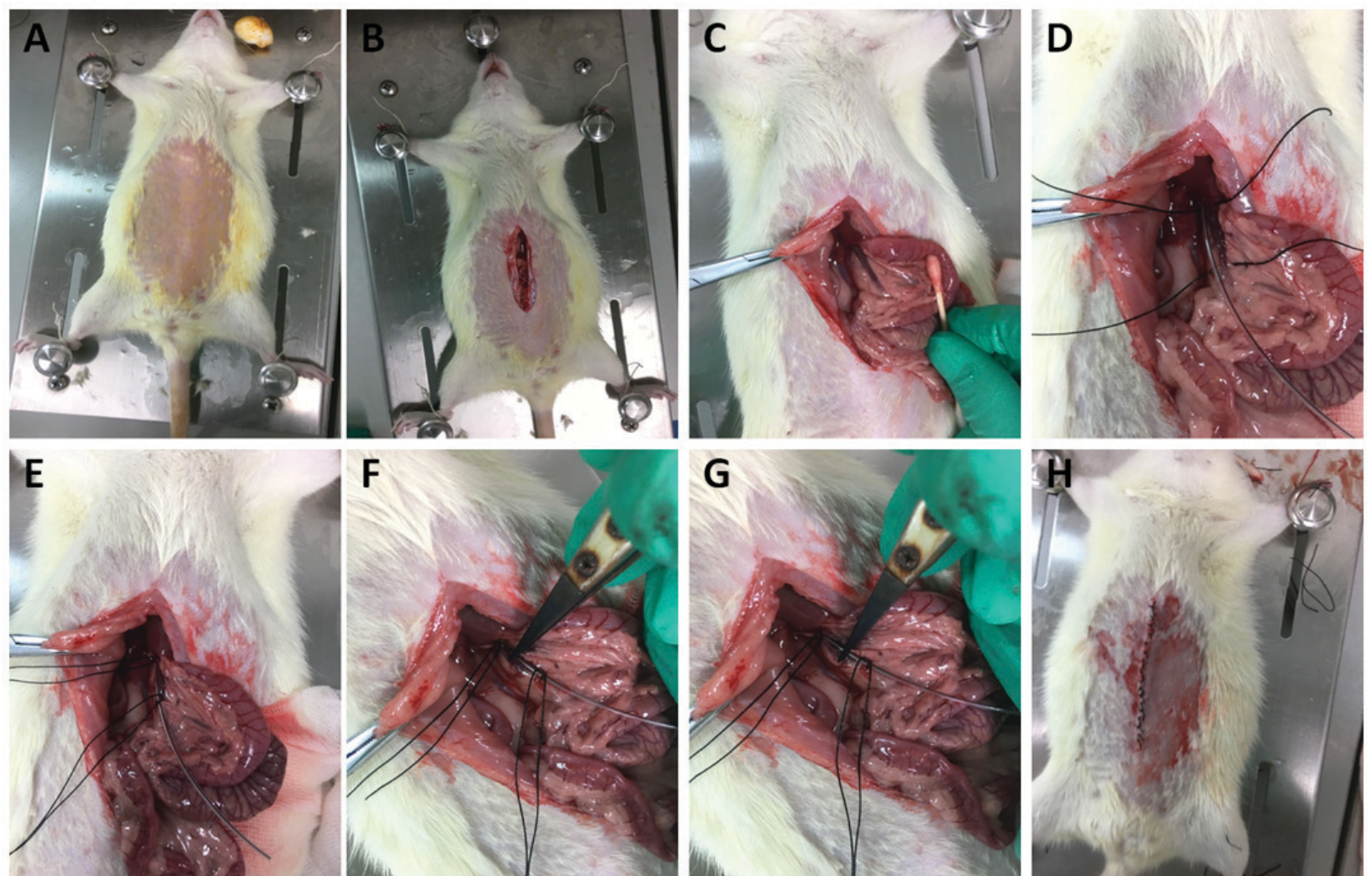

$\mathbf{F}$
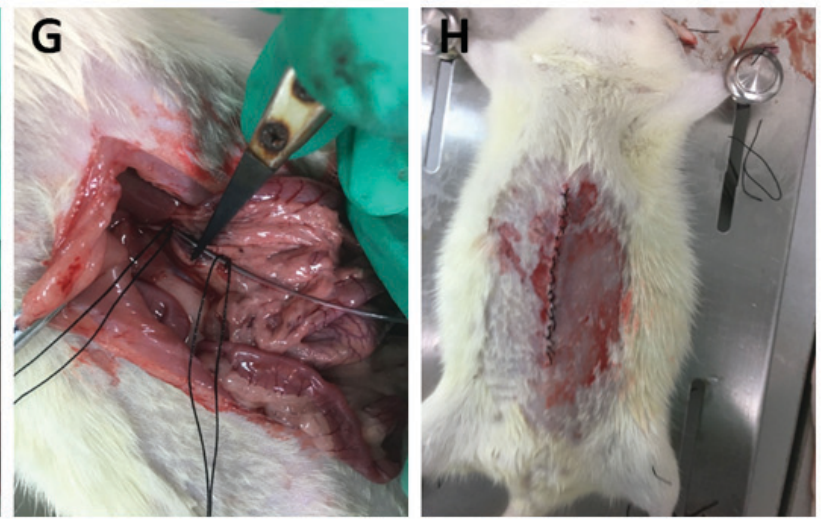

Figure 1. Surgical procedure used to construct the portal vein thrombosis model in rats. (A) Skin preparation and disinfection. (B) A 4-5-cm longitudinal incision. (C) The intestine was partially removed. (D) A section of the portal vein $(1.5-2 \mathrm{~cm})$ was exposed. (E) The proximal and distal ends of the free portal vein were ligated. The portal vein was clamped from $(\mathrm{F})$ the proximal to $(\mathrm{G})$ the distal ends. $(\mathrm{H})$ The abdominal cavity was sutured following surgery.

rPSGL-Ig. Whole blood was collected from the portal vein at $48 \mathrm{~h}$ to detect the mRNA changes in P-selectin by RT-qPCR. Next, the PVT model SD rats ( $n=10 /$ group) were injected with saline, $8 \mathrm{mg} / \mathrm{kg}$ rPSGL-Ig or $2 \times 10^{4} \mathrm{U} / \mathrm{kg}$ urokinase (URO; Livzon Pharmaceutical Group Inc., Zhuhai, China), a commonly used thrombolytic drug (22). The thrombus size pretherapy, and 12, 24, and $48 \mathrm{~h}$ after rPSGL-Ig treatment were observed using a B-mode ultrasound instrument.

Statistical analysis. Quantitative data were expressed as mean \pm standard deviation. The quantitative data between groups were analyzed by a one-way analysis of variance followed by Bonferroni post hoc tests, which were analyzed with SPSS version 17.0 (SPSS, Inc., Chicago, IL, USA). P<0.05 indicated that the difference between groups was statistically significant.

\section{Results}

Thrombi and vessel diameters are larger in the rat model of PVT. A rat model of PVT was successfully constructed (Fig. 1). To examine the stability of the thrombus, the vascular wall with visible thrombus was punctured at 6, 12 and 24-h post-surgery. An unstable red thrombus, which occurred following a hemorrhage, was formed $6 \mathrm{~h}$ after surgery and became enlarged $12 \mathrm{~h}$ after surgery (Fig. 2A and B). At $24 \mathrm{~h}$ after surgery, a stable black thrombus developed, exhibiting a hardened vascular wall and venous stenosis (Fig. 2C). B-Scan ultrasonography demonstrated that the thrombus size and vessel diameter in the model group were significantly increased starting $6 \mathrm{~h}$ post-surgery compared with the control group in what appeared to be a time-dependent manner (all $\mathrm{P}<0.05$; Table I; Fig. 2D-I). Additionally, a 2.5-fold higher expression of P-selectin was observed in the model group compared with the control group $(\mathrm{P}<0.01$; Fig. $2 \mathrm{~J})$.

rPSGL-Ig inhibits PVT formation. The inhibitory effects of rPSGL-Ig on the formation of PVT were evaluated by B-scan ultrasonography. Thrombus size and vessel diameter were increased in the model group (Table I). Intervention with rPSGL-Ig significantly inhibited PVT formation by lowering the thrombus size and vessel diameter at 6-24 h post-surgery compared with the model group (all $\mathrm{P}<0.05$ ).

rPSGL-Ig relieves histopathological changes in the portal vein, central hepatic vein, and vasa intestinae tenuis. Histopathological changes in the portal vein, central hepatic vein and vasa intestinae tenuis in rats of different groups were evaluated by HE staining. A normal structure of endangium with flattened endothelial cells and oriented typical media smooth muscle cells in portal vein was presented in control group (Fig. 3A and B). As shown in Fig. 3C and D, the portal vein in the model group exhibited a damaged endangium, exfoliated endothelial cells and thickened media smooth muscle cells. A thrombus containing an evident fibrin network, platelet trabeculae, adipocytes and leukocytes was observed in the portal vein of the model group. Following intervention with rPSGL-Ig, thrombus formation in the portal vein was markedly 
Table I. Portal vein thrombosis formation detected by B-scan ultrasonography.

\begin{tabular}{llcccccc}
\hline & & & \multicolumn{5}{c}{ Post-operation } \\
\cline { 6 - 8 } Parameter & Group & $\begin{array}{c}30 \mathrm{~min} \\
\text { pre-operation }\end{array}$ & $30 \mathrm{~min}$ & $3 \mathrm{~h}$ & $6 \mathrm{~h}$ & $12 \mathrm{~h}$ & $24 \mathrm{~h}$ \\
\hline Thrombus & Control & 0 & 0 & 0 & 0 & 0 & 0 \\
size $\left(\mathrm{cm}^{2}\right)$ & Model & 0 & 0 & 0 & $0.079 \pm 0.037^{\mathrm{a}}$ & $0.138 \pm 0.035^{\mathrm{a}}$ & $0.204 \pm 0.039^{\mathrm{a}}$ \\
& rPSGL-Ig & 0 & 0 & 0 & $0.049 \pm 0.006^{\mathrm{a}, \mathrm{b}}$ & $0.062 \pm 0.022^{\mathrm{a}, \mathrm{b}}$ & $0.096 \pm 0.025^{\mathrm{a}, \mathrm{b}}$ \\
Vessel & Control & $0.134 \pm 0.009$ & $0.135 \pm 0.011$ & $0.134 \pm 0.009$ & $0.142 \pm 0.009$ & $0.147 \pm 0.020$ & $0.148 \pm 0.010$ \\
diameter $(\mathrm{cm})$ & Model & $0.136 \pm 0.013$ & $0.134 \pm 0.015$ & $0.146 \pm 0.013^{\mathrm{a}}$ & $0.178 \pm 0.033^{\mathrm{a}}$ & $0.194 \pm 0.026^{\mathrm{a}}$ & $0.210 \pm 0.031^{\mathrm{a}}$ \\
& rPSGL-Ig & $0.135 \pm 0.007$ & $0.137 \pm 0.015$ & $0.140 \pm 0.008^{\mathrm{b}}$ & $0.156 \pm 0.009^{\mathrm{b}}$ & $0.168 \pm 0.025^{\mathrm{a}, \mathrm{b}}$ & $0.176 \pm 0.018^{\mathrm{a}, \mathrm{b}}$ \\
& &
\end{tabular}

${ }^{\mathrm{a}} \mathrm{P}<0.05$ vs. Control; ${ }^{\mathrm{b}} \mathrm{P}<0.05$ vs. Model. rPSGL-Ig, recombinant $\mathrm{P}$-selectin glycoprotein ligand immunoglobulin G.

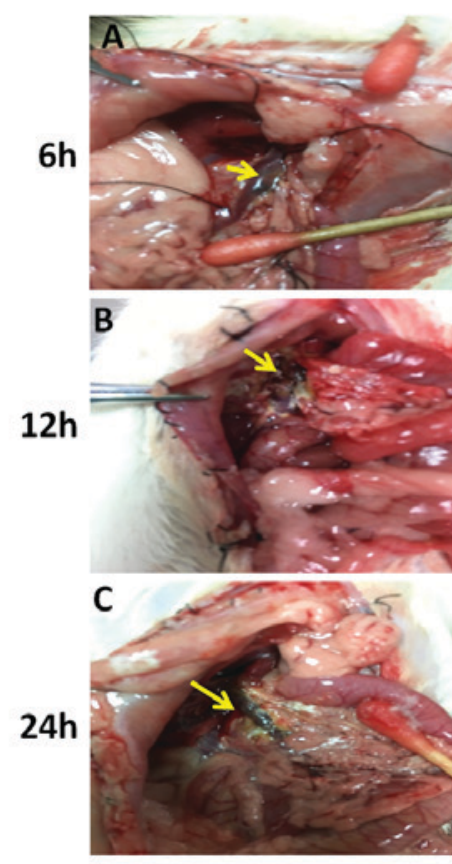

Thrombus
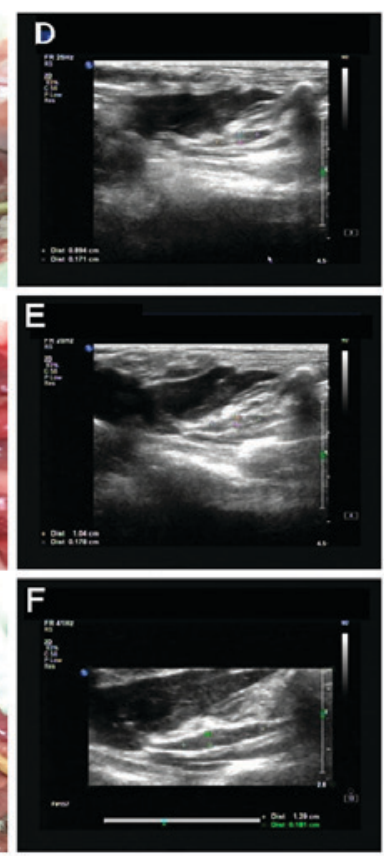

Thrombus size
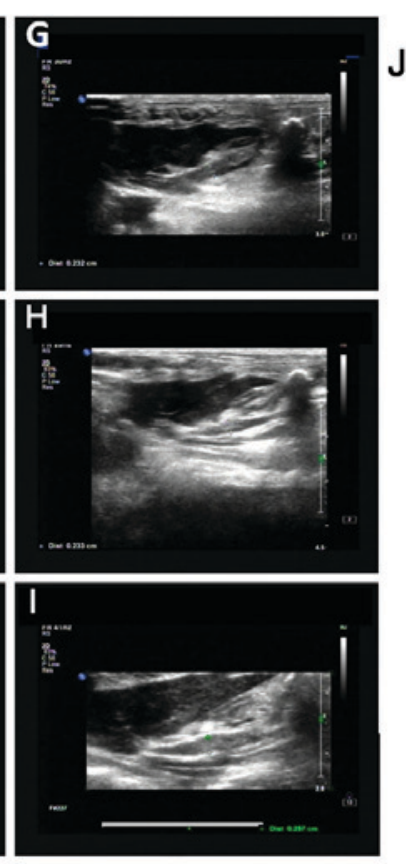

Vessel diameter

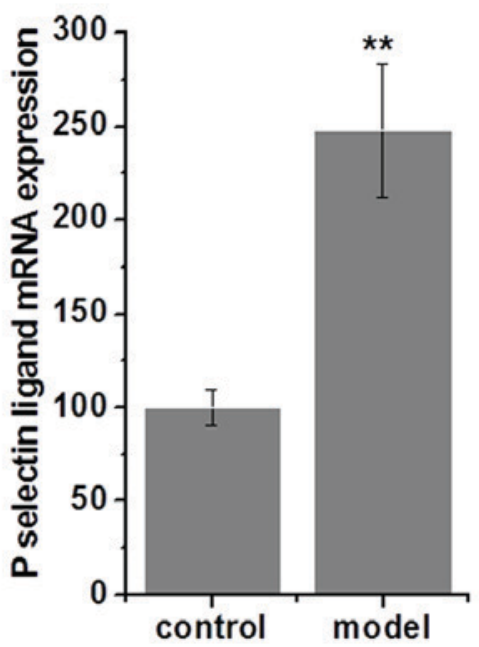

Figure 2. Formation of portal vein thrombosis in the model group. Macro-observation of the thrombus at (A) 6 , (B) 12 and (C) 24 h post-surgery. Evaluation of thrombus size at (D) 6, (E) 12 and (F) 24 h post-surgery. Evaluation of vessel diameter at (G) 6, (H) 12 and (I) 24 h post-surgery. (J) The expression of the $\mathrm{P}$-selectin mRNA in the control and model groups determined by reverse transcription-quantitative polymerase chain reaction. ${ }^{* *} \mathrm{P}<0.01 \mathrm{vs}$. the control group.

inhibited, revealing an imperceptible fibrin network and platelet trabeculae, and reduced adipocytes (Fig. 3E and F). Additionally, erythrocytes were not observed in the central hepatic veins of the control group, which also presented with a compact venous wall structure without cellular swelling (Fig. 4A and B). However, an expanded venous wall, deposited erythrocytes and slightly swollen liver cells were observed in the central hepatic veins of the model group (Fig. 4C and D). Although blood extravasation was also observed in the rPSGL-Ig group, other histopathological changes were markedly relieved (Fig. 4E and F). Furthermore, erythrocytes were not present and normal venous structure with compact intestinal wall structures was observed in the vasa intestinae tenuis of the control group (Fig. 5A and B). Similar histopathological changes were observed in the vasa intestinae tenuis of the model and rPSGL-Ig groups (Fig. 5C-F).
rPSGL-Ig relieves histopathological changes in the portal vein. TEM was performed to evaluate histopathological changes in the portal veins in rats of different groups. As shown in Fig. 6A-C, a normal ultrastructure of the portal vein was observed in the control group with structurally complete granulocytes and vascular endothelial cells. In the model group, massive red blood cell deposition along with destruction of the surrounding histiocytic structure was observed 6-h post-surgery (Fig. 6D). The surrounding histiocytic structure was destroyed and necrosed 12 and 24-h post-surgery, respectively, with numerous cell fragments and a small number of granulocytes remaining (Fig. 6E and F). Following treatment with rPSGL-Ig, there were many deposited erythrocytes and slight thrombosis in the portal veins at 6-h post-surgery, with partial destruction of surrounding veins and histiocytic cell structures (Fig. 6G). There were no obvious changes at 12-h 


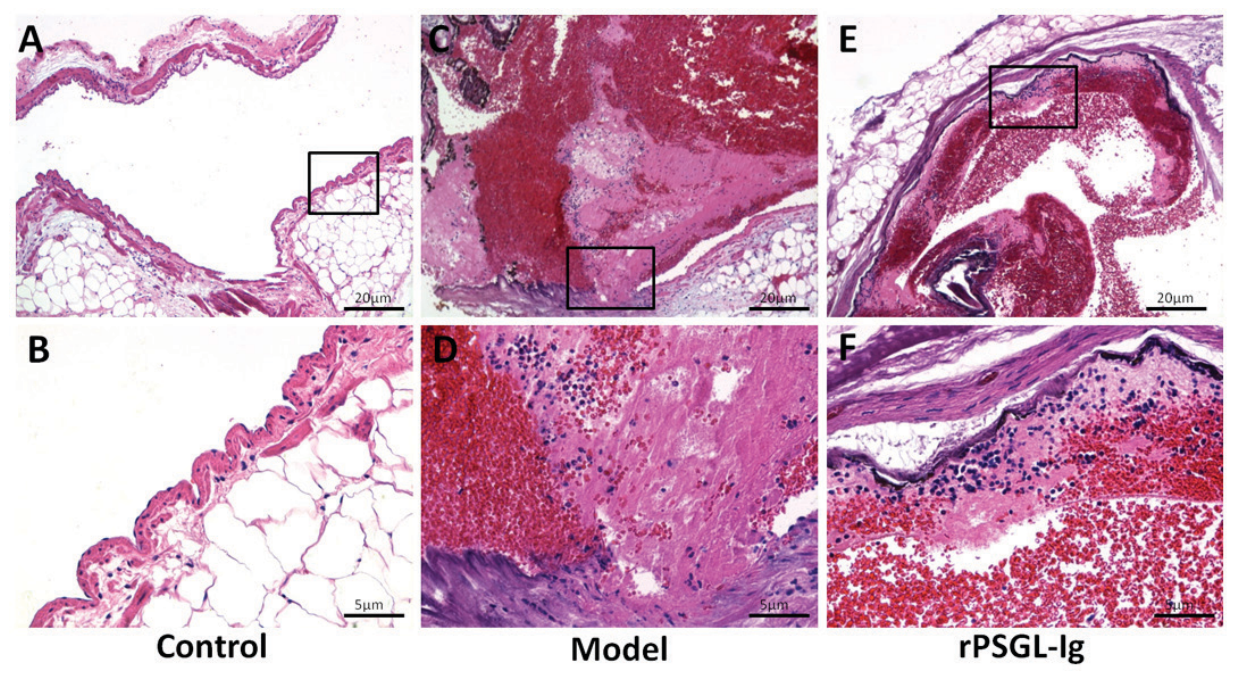

Figure 3. Portal veins were stained with hematoxylin and eosin in the control (A, magnification, $\mathrm{x} 100$; $\mathrm{B}$, magnification, $\mathrm{x} 400$ ), portal vein thrombosis model (C, magnification, x100; D, magnification, x400) and rPSGL-Ig (E, magnification, x100; F, magnification, x400) groups.
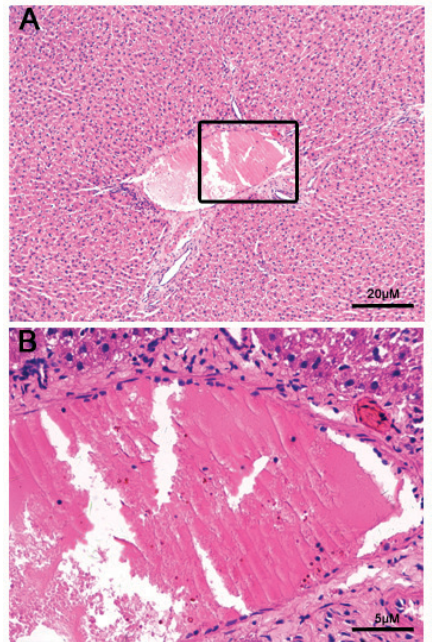

Control
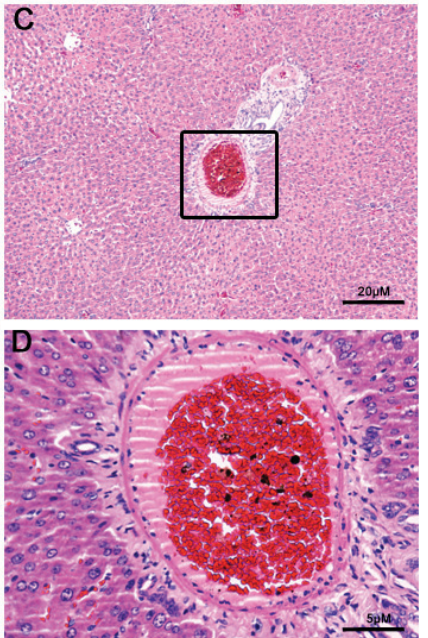

Model
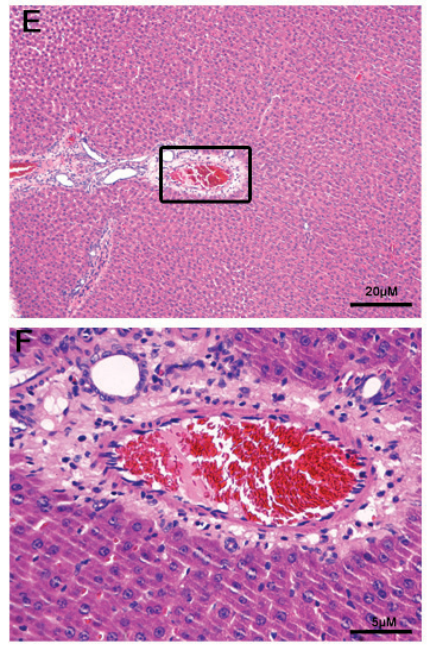

rPSGL-Ig

Figure 4. Central hepatic veins were stained with hematoxylin and eosin in the control (A, magnification, $\mathrm{x} 100$; B, magnification, $\mathrm{x} 400$ ), portal vein thrombosis model (C, magnification, x100; D, magnification, x400) and rPSGL-Ig (E, magnification, x100; F, magnification, x400) groups.
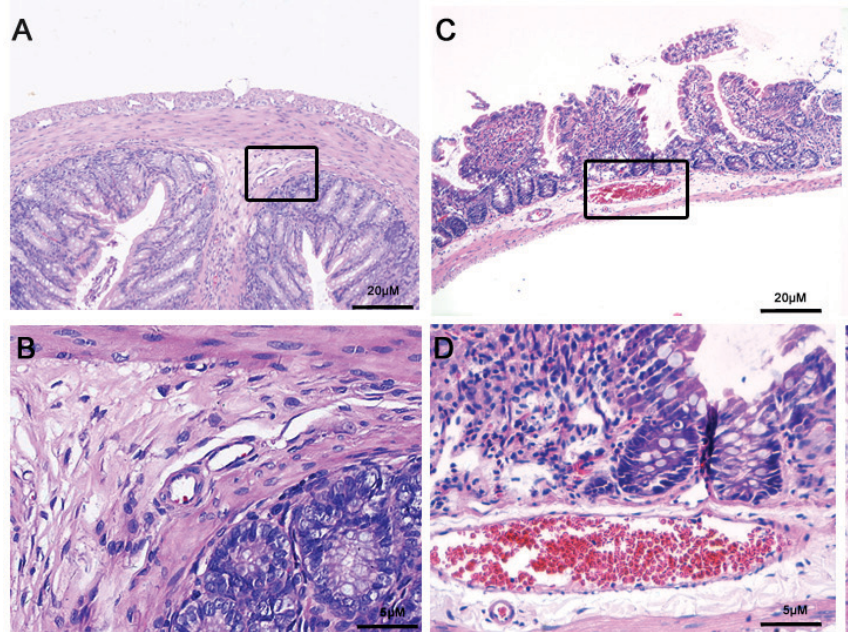

Control

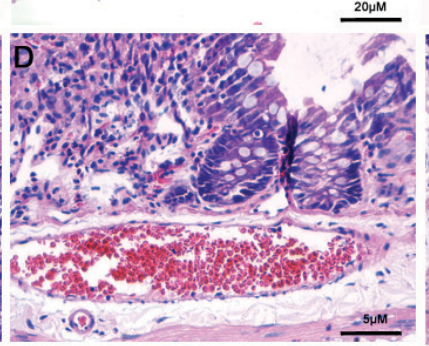

Model
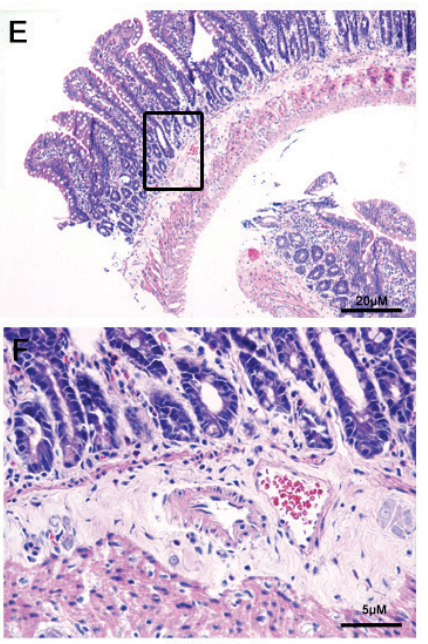

rPSGL-Ig

Figure 5. Vasa intestinae tenuis were stained with hematoxylin and eosin in the control (A, magnification, $\mathrm{x} 100$; B, magnification, $\mathrm{x} 400$ ), portal vein thrombosis model (C, magnification, x100; D, magnification, x400) and rPSGL-Ig (E, magnification, x100; F, magnification, x400) groups. 


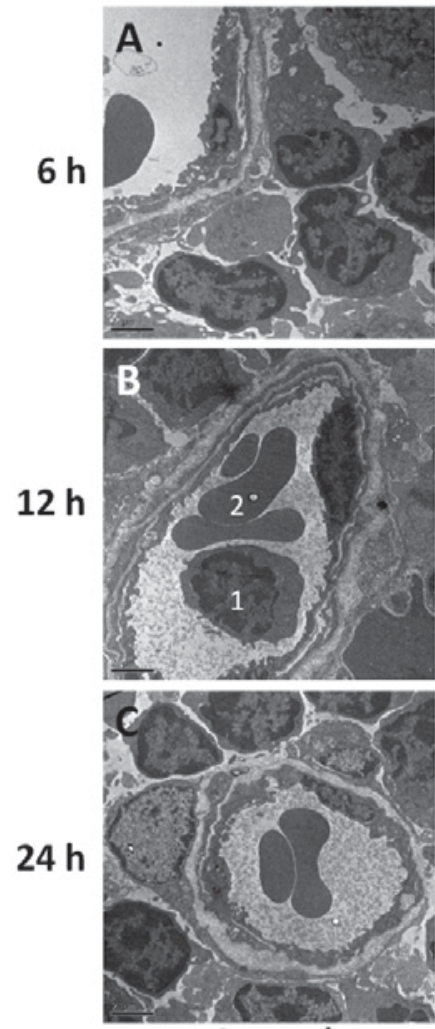

Control
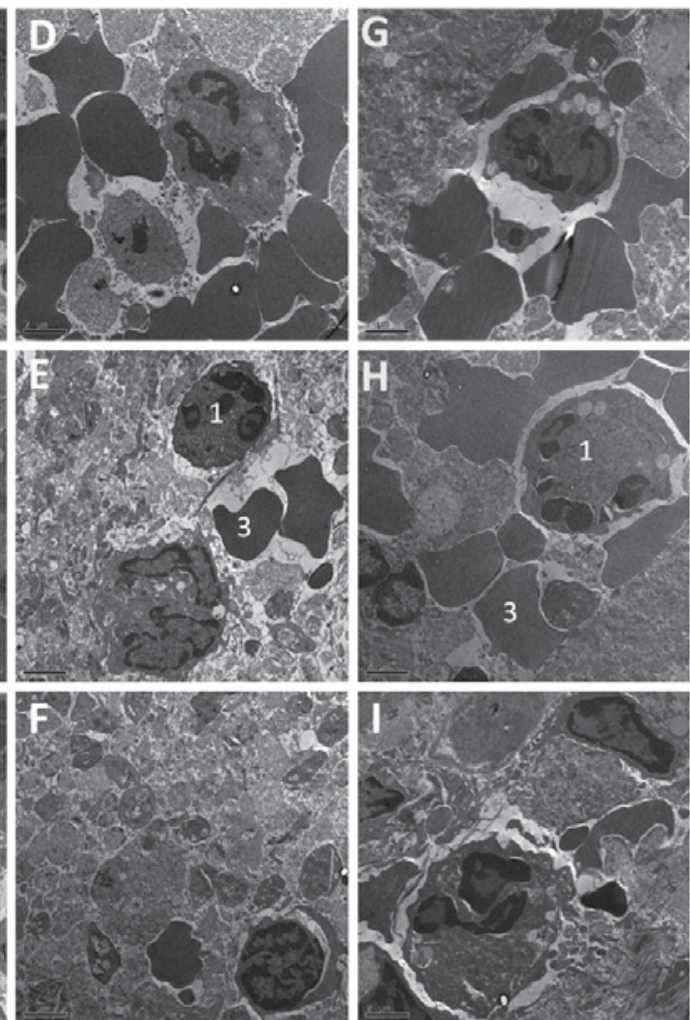

Model

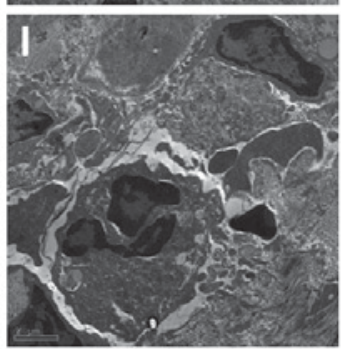

rPSGL-Ig

Figure 6. Transmission electron microscope observation of the portal vein in the control group at (A) 6, (B) 12 and (C) $24 \mathrm{~h}$ post-surgery, the portal vein thrombosis model at (D) 6, (E) 12 and (F) $24 \mathrm{~h}$ post-surgery, and the rPSGL-Ig group at (G) 6, (H) 12 and (I) $24 \mathrm{~h}$ post-surgery. Magnification, x1,000. 1, Granulocyte;

2, vascular endothelial cell; 2, deposited red blood cell; rPSGL-Ig, recombinant P-selectin glycoprotein ligand immunoglobulin G.

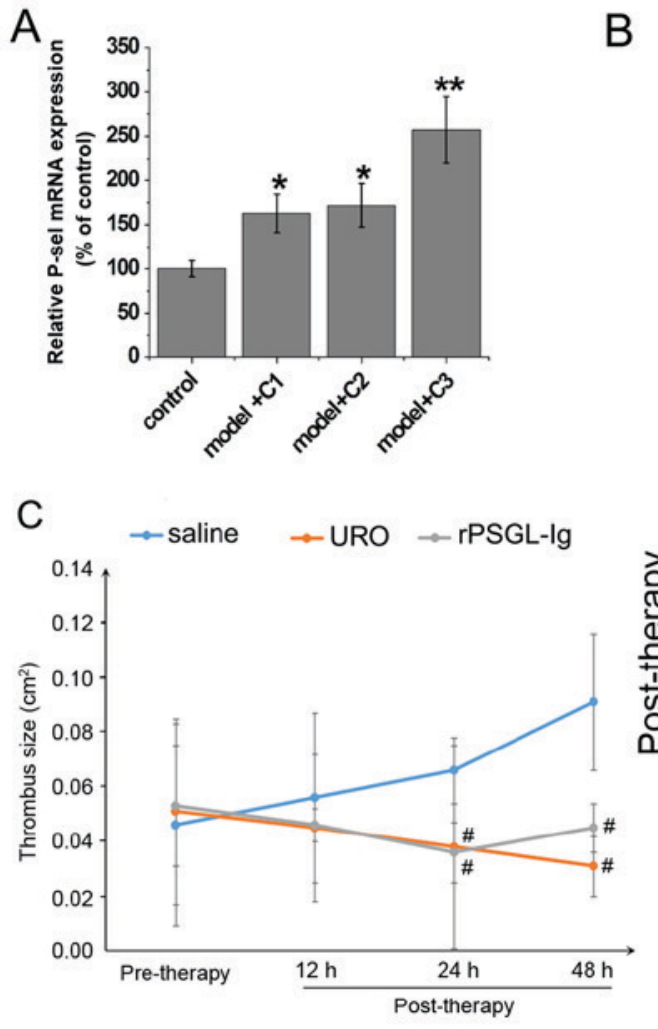

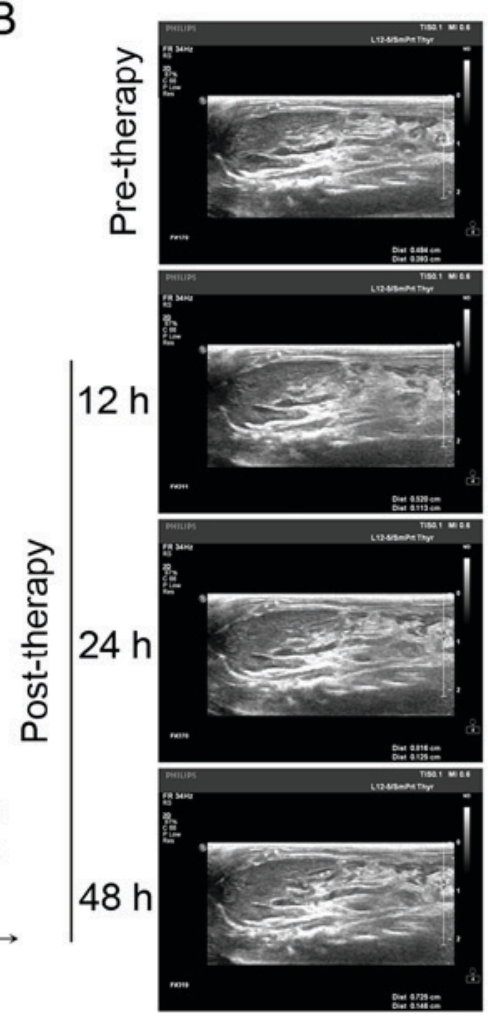

saline

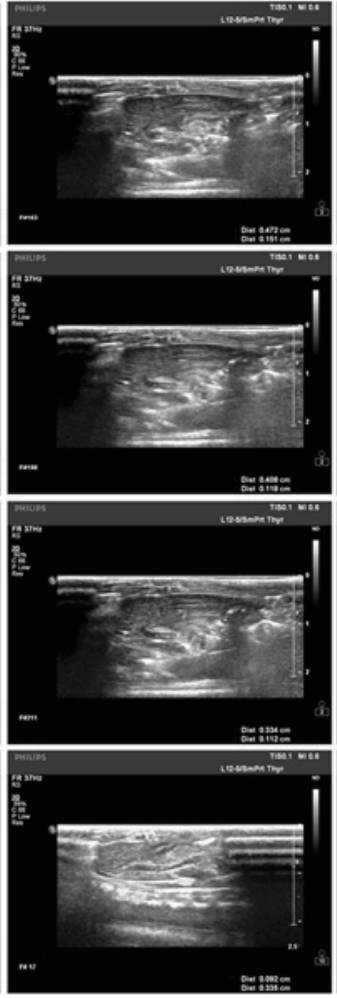

URO
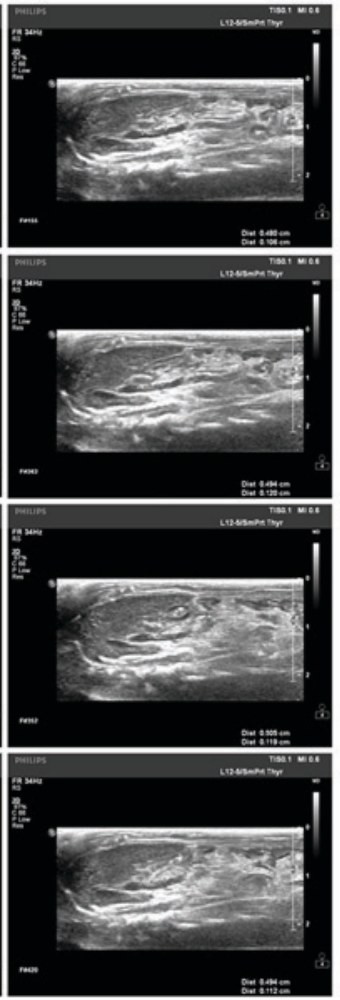

rPSGL-Ig

Figure 7. Thrombolytic effect of rPSGL-Ig. (A) The mRNA expression of P-sel by reverse transcription-quantitative polymerase chain reaction. ${ }^{*}<0.05$ and ${ }^{* *} \mathrm{P}<0.01$ vs. control. (B) Ultrasound images and (C) quantification of thrombus size. ${ }^{\# P}<0.05$ vs. saline. URO, urokinase; rPSGL-Ig, recombinant $\mathrm{P}-$ selectin glycoprotein ligand immunoglobulin G; C1, 4 mg/kg rPSGL-Ig; C2, mg/kg rPSGL-Ig 6; C3, 8 mg/kg rPSGL-Ig; P-sel, P-selectin. 
Table II. Thrombus length in rats following treatment with rPSGL-Ig.

\begin{tabular}{lcccccc}
\hline Group & \multicolumn{7}{c}{ Thrombus length (cm) } \\
\hline Control & 0 & 0 & 0 & 0 & 0 & 0 \\
$4 \mathrm{mg} / \mathrm{kg} \mathrm{rPSGL-Ig}$ & 0.5 & 0.5 & 0.7 & 0.6 & 0.8 & 0.7 \\
$6 \mathrm{mg} / \mathrm{kg} \mathrm{rPSGL-Ig}$ & 0.2 & 0.3 & 0.1 & 0.3 & 0.4 & 0.3 \\
$8 \mathrm{mg} / \mathrm{kg}$ rPSGL-Ig & 0.1 & 0 & 0 & 0 & 0.2 & 0.1 \\
\hline
\end{tabular}

rPSGL-Ig, recombinant P-selectin glycoprotein ligand immunoglobulin G.

post-surgery (Fig. 6H), however, a reduction in the number of erythrocytes deposited and the lack of thrombus in the portal vein, as well as intact surrounding veins and histiocytic cell structures was observed at 24-h post-surgery following treatment with rPSGL-Ig (Fig. 6I).

rPSGL-Ig has thrombolytic effects in the portal vein of $P V T$ rats. Evaluation of the thrombolytic effect of rPSGL-Ig revealed a decreased thrombus length (Table II), while the level of P-selectin mRNA was significantly upregulated compared with the control group $(\mathrm{P}<0.01$; Fig. 7A) at a dose of $8 \mathrm{mg} / \mathrm{kg}$. Thus, $8 \mathrm{mg} / \mathrm{kg}$ rPSGL-Ig was used to detect thrombolytic effects. No significant change in thrombus size in the rPSGL-Ig group was observed, while thrombus size decreased gradually in the URO group with time (Fig. 7B and C). However, the saline group showed an increasing trend. Thrombus size was significantly decreased in the URO and rPSGL-Ig groups compared with the saline group at 24 and 48-h post-surgery (all $\mathrm{P}<0.05)$. The best thrombolytic effect was observed in the URO group followed by the rPSGL-Ig group, while the saline group showed the lowest effects. Therefore, rPSGL-Ig intervention exhibited a thrombolytic effect on PVT rats, however it was less effective than URO.

\section{Discussion}

The current study was initially designed to establish a novel method for constructing a portal vein thrombosis (PVT) model. The specific anatomical characteristics of PVT greatly limit the application of traditional modeling methods. Complete vein ligation is commonly conducted to construct vein thrombosis models $(23,24)$. However, complete ligation of the portal vein may induce intestinal congestion, and even intestinal necrosis and perforation. As the portal vein is the main blood supply to the liver, complete ligation of the portal vein can also lead to hepatic failure. These complications contribute to the animal models succumbing to their injuries. In the current study, a stable PVT model was constructed in rats by IPVO combined with endangium destruction. The proximal and distal ends of the free portal vein were ligated to block blood flow, and then the portal vein was clamped from the proximal to the distal ends to damage the intima. A total of $24 \mathrm{~h}$ post-surgery, stable black thrombi with hardened vascular walls and venous stenosis were formed, supporting the validity of the modeling method. Additionally, since the portal vein is relatively thin, and has confluent intestinal and splenic branches, puncture catheterization is difficult to perform (25). In the current study, B-scan ultrasonography was used to evaluate thrombus formation. The results demonstrated that the thrombus size and vessel diameter in the model group was significantly increased from $6 \mathrm{~h}$ post-surgery in what appeared to be a time-dependent manner. These findings further illustrate that the modeling method is feasible and efficient.

PSGL-1, a high-affinity ligand of P-selectin, serves an important role in thrombus formation (26). PSGL-1 is involved in leukocyte-endothelial and leukocyte-platelet interactions, and contributes to the development of a platelet-rich thrombus following vessel wall injury (26). In the current study, a 4.0-fold higher expression of P-selectin was observed in the model group compared with the control group, demonstrating the procoagulant role of P-selectin in PVT. Studies have revealed that inhibition of the P-selectin signaling pathway is an effective therapeutic target in the thrombus. For example, the P-selectin small-molecule antagonist, PSI-697, has been demonstrated to reduce the thrombus weight by $18 \%$ relative to vehicle by inhibiting the binding of P-selectin to PSGL (27). A deficiency in PSGL protects mice from thrombosis following collagen and epinephrine challenge, resulting in mild thrombocytopenia, less fibrin deposition and a low number of thrombosed blood vessels (28). As a specific antibody for PSGL, rPSGL-Ig can also antagonize P-selectin by competing with PSGL-1 to inhibit thrombosis. The authors of the current study demonstrated that rPSGL-Ig induced upregulation of the P-selectin mRNA, indicating that transcription of SELP was substantially increased when P-selectin was antagonized by rPSGL-Ig. Furthermore, it has been reported that rPSGL-Ig can be used to successfully treat established vein thrombosis with no anticoagulation, thrombocytopenia or wound complications (10). P-Selectin inhibition with rPSGL-Ig decreased vein wall fibrosis and enhanced thrombus resolution in a rat model of deep vein thrombosis (29). However, the specific roles of rPSGL-Ig in PVT are not fully understood. In the current study, the antithrombotic effects of rPSLG-Ig in PVT were evaluated. The results revealed that the thrombus size and vessel diameter were significantly decreased in the rPSGL-Ig group compared with the model group. Additionally, histopathological changes in the portal vein, central hepatic vein and vasa intestinae tenuis in the PVT model were markedly relieved by rPSLG-Ig. These findings are consistent with those of previous studies $(30,31)$ and further illustrate that rPSGL-Ig is an effective agent for preventing PVT. In PVT rats, rPSLG-Ig may inhibit platelet activation in an injured arterial circulation, and the combination of activated platelets and neutrophils through the competitive binding of PSGL with rPSLG-Ig prevent the formation of PVT.

Evaluation of the thrombolytic effect of rPSGL-Ig demonstrated that it was more evident as the dosage increased. URO is a commonly used thrombolytic drug in the clinic (32). In the current study, URO was used as a positive control to evaluate the thrombolytic effect of rPSGL-Ig. The results of the B-scan ultrasonography in portal vein revealed that the thrombus in the rPSGL-Ig group was larger compared with that in the URO group, indicating that the thrombolytic effect was not as good as that of URO. However, compared with the saline group, rPSGL-Ig exhibited a thrombolytic therapy function by 
attenuating thrombus formation. Considering the small sample size of the rats and the concentration range of rPSGL-Ig, the thrombolysis effect of rPSGL-Ig requires further study in future experiments. The results of the current study results suggest that rPSGL-Ig prevents PVT formation and promotes thrombolysis.

In conclusion, a PVT model was successfully constructed in rats by IPVO combined with endangium destruction. Intervention with rPSGL-Ig significantly inhibited PVT formation, the thrombus size and the vessel diameter, and markedly relieved histopathological changes in the portal vein, central hepatic vein and vasa intestinae tenuis. However, application of rPSGL-Ig for preventing PVT is limited in clinical practice. Further studies on the clinical effects of rPSGL-Ig are required.

\section{Acknowledgements}

Not applicable.

\section{Funding}

The current study was supported by the Social Public Technology Research and Development Program from the Science and Technology Department of Hunan Province, China (grant no. 2014C33137), and the General Science and Research Project Program (grant no. 2010YSB08) and the Social Public Research Program (grant no. 2017GY47) from the Science and Technology Bureau of Huzhou City, China.

\section{Availability of data and materials}

The datasets used and/or analyzed during the current study are available from the corresponding author on reasonable request.

\section{Authors' contributions}

YW and JS participated in the design of this study and performed statistical analysis. HS, YW, GC, and WC carried out the study and collected important background information. JZ and LY drafted the manuscript. All authors read and approved the final manuscript.

\section{Ethics approval and consent to participate}

The current study was approved by the Ethics Committee of Huzhou Central Hospital (Huzhou, China).

\section{Patient consent for publication}

Not applicable.

\section{Competing interests}

The authors declare that they have no competing interests.

\section{References}

1. Cohen R, Mallet T, Gale M, Soltys R and Loarte P: Portal vein thrombosis. Case Rep Vasc Med 2015: 823063, 2015.
2. Winslow ER, Brunt LM, Drebin JA, Soper NJ and Klingensmith ME: Portal vein thrombosis after splenectomy. Am J Surg 184: 631-636, 2002.

3. Manzano-Robleda Mdel C, Barranco-Fragoso B, Uribe M and Méndez-Sánchez N: Portal vein thrombosis: What is new? Ann Hepatol 14: 20-27, 2015.

4. Amitrano L, Guardascione MA, Brancaccio V, Margaglione M, Manguso F, Iannaccone L, Grandone E and Balzano A: Risk factors and clinical presentation of portal vein thrombosis in patients with liver cirrhosis. J Hepatol 40: 736-741, 2004.

5. Lixue D, Wujun W, Zhang Y, Sun Z, Haitian $\mathrm{H}$ and Liu Q: Clinical analysis of portal vein thrombosis after splenocaval shunt plus devascularization in treatment of portal hypertension. Chin J Hepatobiliary Surg 16: 353-355, 2010 (In Chinese).

6. Harmanci $\mathrm{O}$ and Bayraktar Y: Portal hypertension due to portal venous thrombosis: Etiology, clinical outcomes. World J Gastroenterol 13: 2535-2540, 2007.

7. Geng JG, Chen M and Chou KC: P-selectin cell adhesion molecule in inflammation, thrombosis, cancer growth and metastasis. Curr Med Chem 11: 2153-2160, 2004.

8. AndréP,Hartwell D, Hrachovinovã I,Saffaripour S and Wagner DD: Pro-coagulant state resulting from high levels of soluble P-selectin in blood. Proc Natl Acad Sci USA 97: 13835-13840, 2000.

9. Myers DD Jr, Schaub R, Wrobleski SK, Londy FJ III, Fex BA, Chapman AM, Greenfield LJ and Wakefield TW: P-selectin antagonism causes dose-dependent venous thrombosis inhibition. Thromb Haemost 85: 423-429, 2001.

10. Myers D, Wrobleski S, Londy F, Fex B, Hawley A, Schaub R, Greenfield $\mathrm{L}$ and Wakefield T: New and effective treatment of experimentally induced venous thrombosis with anti-inflammatory rPSGL-Ig. Thromb Haemost 87: 374-382, 2002.

11. Eppihimer MJ and Schaub RG: P-Selectin-dependent inhibition of thrombosis during venous stasis. Arterioscler Thromb Vasc Biol 20: 2483-2488, 2000.

12. Théorêt JF, Bienvenu JG, Kumar A and Merhi Y: P-selectin antagonism with recombinant p-selectin glycoprotein ligand-1 (rPSGL-Ig) inhibits circulating activated platelet binding to neutrophils induced by damaged arterial surfaces. J Pharmacol Exp Ther 298: 658-664, 2001.

13. Reyers I, Mussoni L, Donati MB and de Gaetano G: Failure of aspirin at different doses to modify experimental thrombosis in rats. Thromb Res 18: 669-674, 1980.

14. Brill A, Fuchs TA, Chauhan AK, Yang JJ, De Meyer SF, Köllnberger M, Wakefield TW, Lämmle B, Massberg S and Wagner DD: Von Willebrand factor-mediated platelet adhesion is critical for deep vein thrombosis in mouse models. Blood 117: 1400-1407, 2011.

15. Nakase H, Heimann A and Kempski O: Alterations of regional cerebral blood flow and oxygen saturation in a rat sinus-vein thrombosis model. Stroke 27: 720-728, 1996.

16. Seyed Mortaz SS, Golfam F, Khalaj AR, Taheri HR and Kholdi N: The effect of distal vein branch ligation in side-to-side arterivenous fistula on the patency rate and complication in one year. Daneshvar Medicine 16: 19-24, 2009.

17. Xu Z, Lioi J, Mu J, Kamocka MM, Liu X, Chen DZ, Rosen ED and Alber M: A multiscale model of venous thrombus formation with surface-mediated control of blood coagulation cascade. Biophys J 98: 1723-1732, 2010.

18. Khalid A, Azeez EA, Bhatti TH and Eshak Y: Transcutanous electric nerve stimulation and deep venous thrombosis. Anesthesia \& Analgesia 86: S12, 1998.

19. Nosaka M, Ishida Y, Kimura A and Kondo T: Time-dependent appearance of intrathrombus neutrophils and macrophages in a stasis-induced deep vein thrombosis model and its application to thrombus age determination. Int J Legal Med 123: 235-240, 2009.

20. Fei Y, Zong GQ, Chen J and Liu RM: Evaluation of the value of d-dimer, P-selectin, and platelet count for prediction of portal vein thrombosis after devascularization. Clin Appl Thromb Hemost 22: 471-475, 2016.

21. Livak KJ and Schmittgen TD: Analysis of relative gene expression data using real-time quantitative PCR and the 2(-Delta Delta C(T)) method. Methods 25: 402-408, 2001.

22. Bing Y, Zhao QH and Yu Z: Effect of urokinase on vein wall remodeling after deep vein thrombosis in rats. Di San Jun Yi Da Xue Xue Bao 32: 1970-1975, 2010 (In Chinese).

23. Kyogashima M, Onaya J, Hara A and Taketomi T: Sulfatide can markedly enhance thrombogenesis in rat deep vein thrombosis model. Glycoconj J 15: 915-922, 1998.

24. NosakaM,Ishida Y,Kimura AandKondoT:Immunohistochemical detection of MMP-2 and MMP-9 in a stasis-induced deep vein thrombosis model and its application to thrombus age estimation. Int J Legal Med 124: 439-444, 2010. 
25. Burcharth F: Percutaneous transhepatic catheterization of the portal venous system. Springer Japan, 1991.

26. Furie B and Furie BC: Role of platelet P-selectin and microparticle PSGL-1 in thrombus formation. Trends Mol Med 10: 171-178, 2004.

27. Bedard PW, Clerin V, Sushkova N, Tchernychev B, Antrilli T, Resmini C, Keith JC Jr, Hennan JK, Kaila N, Debernardo S, et al: Characterization of the novel P-selectin inhibitor PSI-697 [2-(4-chlorobenzyl)-3-hydroxy-7,8,9,10-t etrahydrobenzo[h] quinoline-4-carboxylic acid] in vitro and in rodent models of vascular inflammation and thrombosis J Pharmacol Exp Ther 324: 497-506, 2008.

28. Miszti-Blasius K, Debreceni IB, Felszeghy S, Dezso B and Kappelmayer J: Lack of P-selectin glycoprotein ligand-1 protects mice from thrombosis after collagen/epinephrine challenge. Thromb Res 127: 228-234, 2011.
29. Myers DD Jr, Henke PK, Wrobleski SK, Hawley AE, Farris DM, Chapman AM, Knipp BS, Thanaporn P, Schaub RG, Greenfield LJ and Wakefield TW: P-selectin inhibition enhances thrombus resolution and decreases vein wall fibrosis in a rat model. J Vasc Surg 36: 928-938, 2002.

30. McEver RP: P-selectin and PSGL-1: Exploiting connections between inflammation and venous thrombosis. Thromb Haemost 87: 364-365, 2002.

31. Kneuer C, Ehrhardt C, Radomski MW and Bakowsky U: Selectins-potential pharmacological targets? Drug Discov Today 11: 1034-1040, 2006.

32. Marder VJ and Sherry S: Thrombolytic therapy: Current status. N Engl J Med 318: 1512-1520, 1988.

This work is licensed under a Creative Commons Attribution-NonCommercial-NoDerivatives 4.0 International (CC BY-NC-ND 4.0) License. 